\title{
Descripción morfológica y viabilidad del polen de Nothofagus nervosa (Nothofagaceae)
}

\author{
Morphological description and pollen viability of Nothofagus nervosa (Nothofagaceae)
}

\author{
Luz García ${ }^{\mathrm{a}, \mathrm{b} *}$, Magaly Rivero ${ }^{\mathrm{c}}$, Fernando Droppelmann d \\ *Autor de correspondencia: ${ }^{\text {a }}$ Universidad Austral de Chile, Facultad de Ciencias Forestales y Recursos Naturales, \\ Programa de Doctorado en Ciencias Forestales, luzcecilia29@hotmail.com \\ ${ }^{\mathrm{b}}$ Universidad Técnica de Manabí, Facultad de Ingeniería Agronómica, Campus Experimental La Teodomira, \\ km 13,5 Vía a Santa Ana, Santa Ana, Manabí, Ecuador. \\ ${ }^{\mathrm{c}}$ Cooperativa de Mejoramiento Genético de Chile, Valdivia, Chile. \\ ${ }^{\mathrm{d}}$ Universidad Austral de Chile, Facultad de Ciencias Forestales, Instituto de Bosques y Sociedad, Valdivia, Chile.
}

\begin{abstract}
SUMMARY
Understanding the biology of pollen is necessary to optimize the reproduction of plants and the successful implementation of conservation and breeding programs. In order to have information on the morphological characteristics and pollen viability in Nothofagus nervosa, male flowers of trees established in a clonal seed orchard were collected. Morphological characterization of pollen was carried out from observations under light microscope and scanning electron microscope. Motic Images Plus 2.0 software was used for measurements. Viability was assessed by in vitro germination, germination in vivo and staining with p-phenylenediamine. The pollen size of these species ranges from medium to large and the shape is from sub-oblate to oblate spheroid. Initial viability by in vitro germination ranged from 17.1 to $84.9 \%$; with the staining method, it ranged from 70.7 to $99.3 \%$; using in vivo germination it was calculated between 77.4 and $97.1 \%$. There was a strong correlation between in vitro germination and in vivo germination, but there was no correlation between viability calculated by the staining method and that calculated by germination in vivo; whereby the in vitro method is recommended to assess both viability and longevity of pollen of this species. According to assessments by in vitro germination, pollen lost its viability within a period of eight days stored at room temperature.
\end{abstract}

Key words: viability, morphology, longevity, germination, staining.

\section{RESUMEN}

Entender la biología del polen es necesario para mejorar la reproducción de las especies vegetales y para la implementación exitosa de programas de mejoramiento genético y conservación. Con el fin de contar con información sobre las características morfológicas y de viabilidad del polen en Nothofagus nervosa, se colectó flores masculinas de árboles establecidos en un huerto semillero de clones. La caracterización morfológica del polen se realizó a partir de observaciones en microscopio de luz y electrónico de barrido. Para las mediciones se utilizó el software Motic Images Plus 2,0. El polen de esta especie es de tamaño mediano a grande, de forma sub-oblada a oblada esferoidal. La viabilidad se evaluó mediante germinación in vitro, germinación in vivo y tinción con p-fenilendiamina. Su viabilidad inicial, mediante la germinación in vitro varió desde 17,1 a 84,9\%; con el método de tinción osciló de 70,7 a 99,3\% y mediante germinación in vivo se calculó entre 77,4 y 97,1 \%. Hubo una fuerte correlación entre la germinación in vitro y la germinación in vivo, más no hubo correlación entre la viabilidad calculada por el método de tinción y la calculada mediante germinación in vivo, por lo cual se recomienda el método in vitro para realizar evaluaciones tanto de viabilidad como de longevidad del polen de esta especie. Según evaluaciones mediante germinación in vitro, el polen perdió su viabilidad dentro de un lapso de ocho días, almacenado bajo condiciones ambientales $\left( \pm 20^{\circ} \mathrm{C} \mathrm{y} \pm 40 \% \mathrm{HR}\right)$.

Palabras clave: viabilidad, morfología, longevidad, germinación, tinción.

\section{INTRODUCCIÓN}

Los estudios sobre los diferentes aspectos de la biología del polen son considerados como una prioridad para entender la reproducción de las especies vegetales. Además, son indispensables en la implementación exitosa de programas de mejoramiento genético y conservación, para mejorar la eficiencia de los cruzamientos en la reproduc- ción y asegurar una abundante producción de semillas (Dafni y Firmage 2000).

La biología del polen de Nothofagus nervosa (Phil.) Krasser, especie anemófila de la familia Nothofagaceae, considerada un importante componente de los bosques lluviosos templados de Sudamérica Occidental, no ha sido estudiada detalladamente. Se han realizado estudios sobre la distribución natural y variabilidad genética de esta espe- 
cie (Donoso et al. 2006, Gallo et al. 2006, Sabatier et al. 2011) y actualmente también existe interés en diferentes temáticas de su biología reproductiva (García et al. 2013, Torres y Puntieri 2013).

El polen maduro presenta una morfología bien definida que, por lo general, permite la identificación de la especie de la cual procede; por esta razón, la identificación de los caracteres polínicos es de gran importancia en taxonomía y filogenia. En general, los caracteres morfológicos son importantes en cualquiera de las aplicaciones que tiene el estudio del grano de polen; tanto la forma como el tamaño son considerados caracteres de valor taxonómico, pues en general, permanece constante dentro de una misma especie. Su heterogeneidad en un taxón concreto puede ser indicativo de diferentes estados de madurez u origen híbrido, situación que ha sido reportada entre diferentes especies del género Nothofagus (Donoso y Atienza 1984, Donoso et al. 1990, Stecconi et al. 2004).

Los granos de polen de las distintas especies de la familia Nothofagaceae, son básicamente similares entre sí (Cranwell 1939, Heusser 1971). No obstante, las pocas diferencias han permitido inferir la relación filogenética entre las especies de la familia e incluso subdividir al género en cuatro subgéneros (Brassospora, Nothofagus, Fuscospora y Lophozonia), según cuatro tipos polínicos (brassii, fusca A, fusca B y menziesii) definidos en base a características específicas de forma y tamaño de los granos de polen (Dettmann et al. 1990, Hill y Jordan 1993, Manos 1997). En Chile y Argentina se ha determinado el tipo polínico menziessii para especies del género Nothofagus (Cranwell 1939, Auer et al. 1955, Heusser 1971, Veblen et al. 1996).

La viabilidad y germinabilidad del polen depende de factores medioambientales, tales como fotoperiodo, irradiación, temperatura, humedad relativa o alguna interacción entre estos factores (Cecich 1997). También, condiciones ambientales no idóneas en la planta madre, como polución y $\mathrm{pH}$ alto, podrían inhibir la germinación del polen (Bellani y Paoletti 1992). La longevidad en las diferentes especies ha sido relacionada con la pérdida de agua y un prolongado estado de deshidratación, tanto en la naturaleza como en laboratorio (Heslop-Harrison y Heslop-Harrison 1985, Lisci et al. 1994). Aunque no se han realizado estudios acerca de la viabilidad y longevidad del polen de $N$. nervosa, existen antecedentes en otras especies de este género (Rivero et al. 1995, Báez et al. 2002) que constituyen un buen aporte en esta temática.

Dado que existen antecedentes generales sobre la morfología del $N$. nervosa y de otras especies de la misma familia; el presente estudio tuvo como objetivos proveer una descripción detallada del polen de diferentes individuos de la especie para determinar la variabilidad intraespecífica de esta característica. Además, dado el interés en el programa de mejoramiento genético por realizar cruzamientos entre individuos sobresalientes, se estudió la viabilidad y longevidad del polen en varios clones de esta especie establecidos en el huerto semillero Huillilemu, al mismo tiempo se propuso determinar el método idóneo para evaluar la viabilidad del polen.

La hipótesis planteada es que los granos de polen de diferentes individuos de $N$. nervosa tienen una forma característica, pero difieren en el tamaño. Además se planteó que los granos de polen de diferentes individuos de la especie presentan diferentes porcentajes de viabilidad y una corta longevidad.

\section{MÉTODOS}

El polen evaluado se colectó de 16 clones de $N$. nervosa (seis para análisis morfológico y diez para estudio de viabilidad), establecidos en el huerto semillero clonal Huillilemu, localizado en la comuna San José de la Mariquina, a $65 \mathrm{~km}$ de la ciudad de Valdivia, región de Los Ríos, Chile. Esta comuna ocupa una posición de depresión intermedia, donde los inviernos son fríos con un régimen de heladas moderado y veranos relativamente frescos. Las temperaturas varían entre una máxima de 23 a $26{ }^{\circ} \mathrm{C}$ en enero y una mínima de 2,8 a $2,9{ }^{\circ} \mathrm{C}$ en julio. La precipitación media anual es de 1.300 a $1.800 \mathrm{~mm}$. Acumula anualmente entre 865 y 1.131 grados días de desarrollo y entre 2.100 a 2.400 horas de frío (Pérez y Ponce 2012).

El polen utilizado para los diferentes análisis fue obtenido entre septiembre y noviembre del 2012. La recolección de flores masculinas se realizó entre las 11:00 y 14:00, en el estado de anteras maduras, según patrón fenológico de García et al. (2013), ubicadas a una altura aproximada a 1,50 m del suelo, en los árboles seleccionados para el efecto.

Para la caracterización morfológica del polen se realizaron observaciones y capturas fotográficas, tanto en microscopía de luz como en microscopía electrónica de barrido. Para los dos tipos de microscopía se efectuaron observaciones en 100x, 400x y 1000x. Para la descripción morfológica se utilizó la terminología de Erdtman (1952). El origen de los seis clones evaluados, se detalla en el cuadro 1 .

Las mediciones de los granos de polen se realizaron sobre fotografías capturadas con aumento 400x en microscopio Nikon Eclipse E600, utilizando el programa Motic Images Plus 2,0. Para lograr un mejor contraste en las fotografías, se tiñó los granos de polen utilizando azul de anilina y un tinte doble, a base de lignina rosa y carmín acético (figura 1). Se capturaron al menos 30 imágenes fotográficas de granos de polen de cada uno de los clones, y se midieron entre 43 y 153 granos de polen por parámetro y clon. Los caracteres considerados fueron: 1) número, posición y tipo de aberturas, 2) índice del área polar (largo/ ancho en vista polar), 3) tamaño (longitud del eje polar), 4) forma del polen (eje polar (P) /diámetro ecuatorial (E) en vista ecuatorial).

Se evaluó la viabilidad inicial del polen utilizando el método directo de germinación in vivo y los métodos indirectos: germinación in vitro y tinción con el reactivo pfenilendiamina. Se realizó esta evaluación mediante estos 
Cuadro 1. Código y origen de los árboles donantes del polen evaluado en la caracterización morfológica.

Code and origin of pollen donor trees evaluated in the morphological characterization.

\begin{tabular}{clccc}
\hline Código & Lugar de origen & Altitud (m s.n.m.) & Latitud S & Longitud O \\
\hline 38 & Arquilhue & 540 & $40^{\circ} 07^{\prime}$ & $72^{\circ} 02^{\prime}$ \\
11 & Neltume & 620 & $39^{\circ} 49^{\prime}$ & $71^{\circ} 58^{\prime}$ \\
19 & Neltume & 700 & $39^{\circ} 51^{\prime}$ & $71^{\circ} 53^{\prime}$ \\
21 & Pirihueico & 840 & $40^{\circ} 00^{\prime}$ & $71^{\circ} 41^{\prime}$ \\
30 & Pilmaiquén & 1.000 & $39^{\circ} 54^{\prime}$ & $71^{\circ} 51^{\prime}$ \\
26 & Maihue & $952^{*}$ & $40^{\circ} 16^{\prime}$ & $71^{\circ} 58^{\prime}$ \\
\hline
\end{tabular}

"Esta información de altitud de la localidad de Maihue no está registrada en los archivos de la Cooperativa de Mejoramiento Genético de Chile, se obtuvo en el Google Earth.

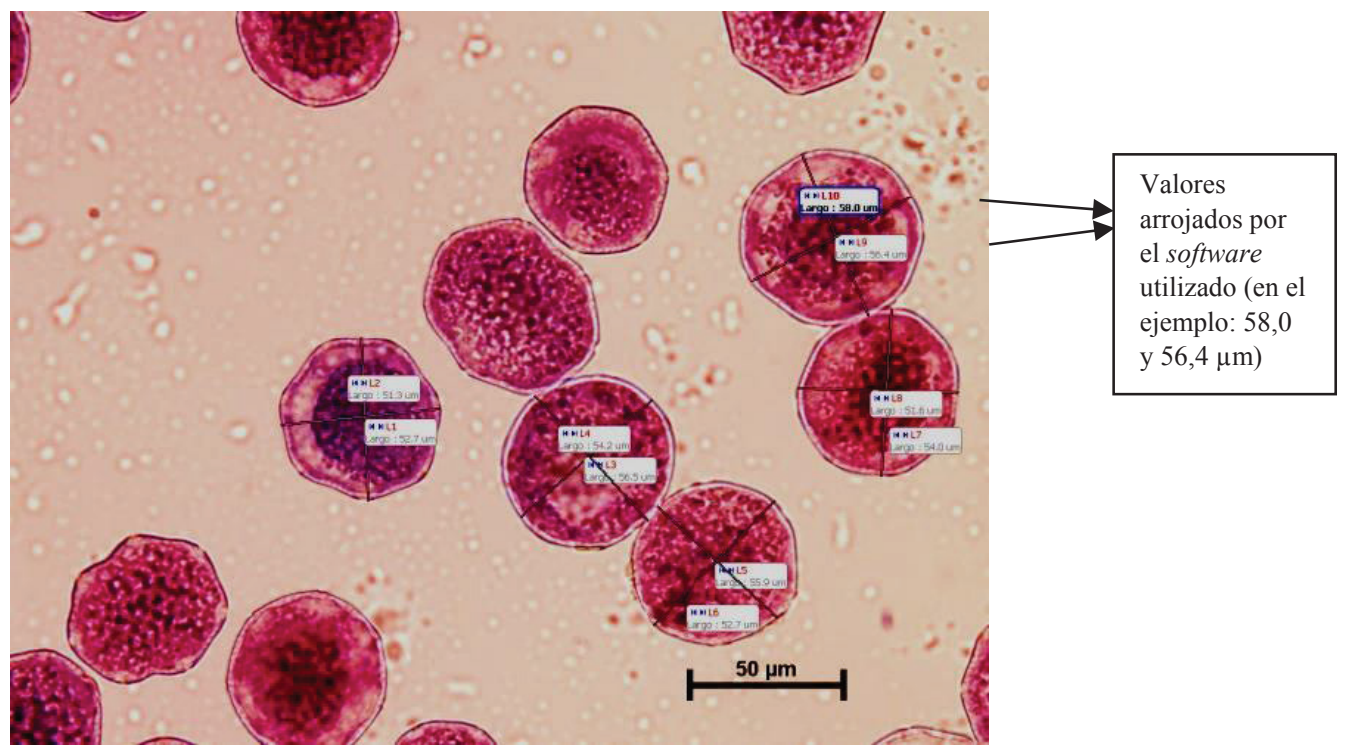

Figura 1. Mediciones polen de Nothofagus nervosa mediante el software Motic Images Plus 2,0.

Measurements of pollen of Nothofagus nervosa by Motic Images Plus 2.0 software.

tres métodos para poder comparar los dos métodos indirectos con el método de germinación in vivo, puesto que se ha demostrado que el número de tubos polínicos que crecen en el estilo es el mejor indicador de la eficacia de una muestra de polen en la inducción de la fructificación y producción de semillas (Shivanna y Rangaswamy 1992).

Para evaluar la germinación in vitro se utilizó polen fresco y el medio estuvo compuesto de sacarosa $(16 \%)$ y agar $(0,4 \%)$ (determinado como el más adecuado en ensayos previos). Este polen se sembró el mismo día de la colecta y se mantuvo en incubación durante 24 horas a $20^{\circ} \mathrm{C}$. El número de granos de polen germinados se determinó bajo un microscopio óptico; considerando un grano de polen como germinado, cuando el tubo polínico tenía una longitud igual o superior al diámetro del grano de polen.

Para calcular la germinación in vivo se realizó polinización manual, utilizando polen fresco de individuos coespe- cíficos. Para esto, previamente se habían aislado tres ramas con brotes florales, por árbol, antes de la antesis, para asegurar que los estigmas no recibieran polen de otros individuos. Esta polinización se realizó cuando las inflorescencias femeninas se encontraban en la fenofase de estigmas receptivos. La receptividad del estigma se determinó mediante observación directa de las flores, según el patrón fenológico de la floración establecido por García et al. (2013).

El desarrollo del tubo polínico se determinó 72 horas después de la polinización, utilizando la metodología propuesta por Shivanna y Rangaswamy (1992): se colectaron cinco inflorescencias por rama, que fueron colocadas en placas Petri con agar para su transporte al laboratorio, donde fueron almacenadas en congelación hasta su evaluación. Para las observaciones, se cortaron los estigmas y se los ablandó sumergiéndolos en $\mathrm{NaOH} 8 \mathrm{~N}$ durante seis horas, luego se aplastaron sobre un porta-objetos y se tiñeron 
con azul de anilina en lactofenol ( $0,1 \%)$. La evaluación de los granos de polen germinados por estigma, se realizó bajo microscopio óptico. Se consideró un grano de polen como germinado cuando había emitido tubo polínico en contacto con la superficie del estigma.

Se evaluó la viabilidad del polen por el método indirecto de tinción, utilizando el reactivo p-fenilendiamina, según el protocolo descrito por Rodríguez-Riano y Dafni (2000). Para la tinción de los granos de polen, se calentó una pequeña cantidad de la solución a $37{ }^{\circ} \mathrm{C}(10 \mathrm{a}$ $15 \mathrm{~min}$ ) y se aplicó por gotas a un grupo de granos de polen colocados sobre un portaobjetos. La cuantificación del número de granos de polen viables se realizó bajo microscopio óptico, considerando como granos de polen viables los que se tornaban de pardo oscuro a negros.

La longevidad del polen se evaluó, cada 48 horas, mediante germinación in vitro (utilizando el medio antes señalado), hasta que la germinabilidad del polen descendió a menos del $5 \%$. Durante el tiempo de evaluación, el polen fue almacenado en tubos Eppendorf (sin pasar por ningún proceso de secado), bajo condiciones ambientales $\left( \pm 20^{\circ} \mathrm{C}\right.$ $\mathrm{y} \pm 40 \% \mathrm{HR}$ ). Esta evaluación se realizó en polen de cinco árboles, cuyo origen y códigos se detallan en el anexo 1.

Análisis estadístico. Para los datos de tamaño se calculó la media, desviación estándar y valores mínimos y máximos. La comparación entre medias se realizó usando la prueba no paramétrica de Kruskal Wallis $(P \leq 0,01)$, puesto que según la prueba de Shapiro-Wilk los datos no seguían una distribución normal.

En los tres ensayos de viabilidad, la variable a evaluar fue el número de granos de polen viables (germinados o teñidos), expresado en porcentaje, en función del total de granos de polen. Cada muestra se repitió tres veces, y en cada placa se evaluaron cuatro campos de observación de $1,5 \mathrm{~mm}^{2}$ aproximadamente, utilizando un aumento de 100x. Se tomó en cuenta que en cada campo de observación existieran un mínimo de 100 granos de polen, lo que significa que se evaluó un mínimo de 1.200 granos de polen por muestra.

Se elaboraron gráficos de dispersión y se calcularon los coeficientes de correlación lineal, previa homogenización de los datos (prueba de Barlet), para determinar el grado de asociación entre la germinación in vitro y germinación in vivo y entre la viabilidad por el método de tinción y la viabilidad calculada mediante germinación in vivo. Además, los valores promedios de viabilidad inicial del polen, en los diferentes individuos, se contrastaron mediante la prueba de Krustall Wallis $(0,05 \%)$. Todos los análisis se realizaron utilizando el software Statistica (versión 7).

\section{RESULTADOS}

Morfología del polen. Los granos de polen de N. nervosa son isopolares, radiosimétricos, mónadas, microespinulados, colpoaperturados, amb circular a semi-angular (figura 2). En vista ecuatorial, según la relación polar/ecuatorial (P/E) son sub-oblatos $(0,80-0,85 \mu \mathrm{m})$ y oblatos esferoidales $(0,88 \mu \mathrm{m})$ (cuadro 2). En vista polar son poligonales, su índice del área polar es de $0,93 \mu \mathrm{m}$ (cuadro 3 ).

Tamaño del polen. La comparación de medias, utilizando la prueba de Kruskal Wallis $(P \leq 0,01)$ (cuadro 2 y 3 ), reveló diferencias significativas en el tamaño de los granos de polen entre algunos de los individuos analizados. En la vista ecuatorial, el eje ecuatorial, presentó una media de $49,76 \mu \mathrm{m}( \pm 3,29)$, su rango varía entre los diferentes individuos analizados, siendo el valor mínimo de $39,70 \mu \mathrm{m}$ y el máximo de $60,30 \mu \mathrm{m}$ en los individuos 19 y 26 res-

Cuadro 2. Mediciones del polen de Nothofagus nervosa en vista ecuatorial $(\mu \mathrm{m})$. Se indica relación polar/ecuatorial (P/E).

Measurements of Nothofagus nervosa pollen in equatorial view $(\mu \mathrm{m})$. Polar/Equatorial ratio $(\mathrm{P} / \mathrm{E})$ is indicated.

\begin{tabular}{cccccccccccc}
\hline \multirow{2}{*}{$\begin{array}{c}\text { Código } \\
\text { árboles }\end{array}$} & $\mathrm{n}$ & \multicolumn{9}{c}{ Eje polar (P) } & \multicolumn{7}{c}{ Eje ecuatorial (E) } & \multirow{2}{*}{ Forma } \\
\cline { 3 - 9 } & & $\bar{x}^{\mathrm{a}}$ & $\sigma$ & Mínimo & Máximo & $\bar{x}^{\mathrm{b}}$ & $\sigma$ & Mínimo & Máximo & $\mathrm{P}^{\mathrm{c}} \mathrm{E}^{\mathrm{c}}$ & \\
\hline 19 & 71 & $38,93 a$ & 3,42 & 31,5 & 47,7 & $45,67 \mathrm{a}$ & 3,19 & 39,7 & 51,8 & $0,85 a$ & Sub-oblato \\
11 & 68 & $39,31 a$ & 2,54 & 34,8 & 44,9 & $48,82 b$ & 3,00 & 43,2 & 55,0 & $0,81 a$ & Sub-oblato \\
21 & 90 & $40,04 a b$ & 2,86 & 35,9 & 46,2 & $49,57 b c$ & 2,36 & 44,5 & 54,5 & $0,81 a$ & Sub- oblato \\
26 & 50 & $41,03 a b$ & 3,73 & 35,2 & 49,2 & $51,50 b c$ & 4,19 & 44,6 & 60,3 & $0,80 a$ & Sub-oblato \\
30 & 55 & $43,93 b c$ & 4,65 & 38,3 & 51,1 & $50,17 b c$ & 3,25 & 40,7 & 54,9 & $0,88 a$ & Oblato esferoidal \\
38 & 56 & $46,66 c$ & 5,16 & 35,6 & 56,4 & $52,81 c$ & 3,74 & 45,7 & 59,3 & $0,88 a$ & Oblato esferoidal \\
$\bar{x}$ & 41,7 & - & 4,65 & - & - & 49,76 & 3,29 & - & - & 0,84 & \\
\hline
\end{tabular}

Letras diferentes indican medias diferentes, prueba de Kruskal Wallis $\mathrm{H}=67,71, P \leq 0,0000$

Letras diferentes indican medias diferentes, prueba de Kruskal Wallis $\mathrm{H}=75,09, P \leq 0,0000$.

Letras iguales indican medias iguales, prueba de Kruskal Wallis $\mathrm{H}=0,0000, P=1,000$. 
pectivamente; el eje polar osciló entre $31,50 \mu \mathrm{m}$ (clon 19) y 56,40 $\mu \mathrm{m}$ (clon 38), siendo la media de 41,65 $\mu \mathrm{m}$ $( \pm 3,73)$ (cuadro 2). En vista polar, el ancho se calculó en $46,53 \mu \mathrm{m}( \pm 2,45)$ con valores máximo de $60,70 \mu \mathrm{m}$ (clon 38) y mínimo de 38,00 $\mu \mathrm{m}$ (clon 19) (cuadro 3), mientras el largo promedio se calculó en 50,02 $\mu \mathrm{m}( \pm 2,76)$.

Aperturas. Las aperturas son tipo colpos (figura 2), se presentan de seis a nueve en los diferentes individuos, siendo más común los granos de polen con siete aperturas (55,73\%) y los granos de polen menos comunes son los que tienen nueve aperturas $(1,77 \%$ ) (cuadro 4). Según el número, posición y tipo de aperturas, el polen de $N$. nervosa correspondería al tipo polínico poli-zono-colpados.

Viabilidad inicial y longevidad del polen. Los individuos evaluados presentaron porcentajes de germinación inicial entre 17,1 y $84,9 \%( \pm 4,03-19,73)$, resultando en una me-
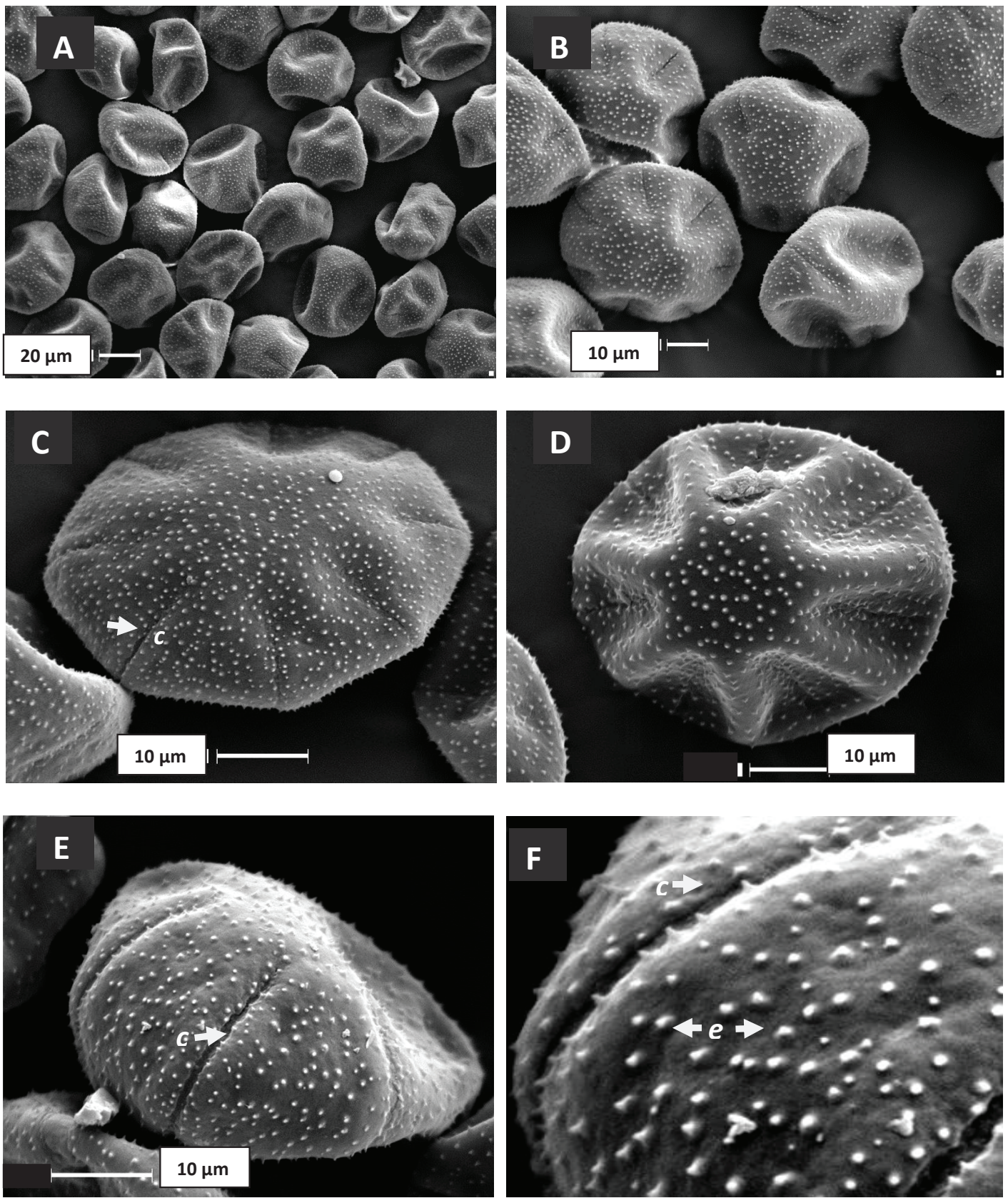

Figura 2. Microfotografías de granos de polen de Nothofagus nervosa en microscopio electrónico de barrido: A y B grupo de granos de polen, C y D vista polar, E vista ecuatorial, F colpo (c) y microespínulas (e).

Photomicrographs of pollen grains of Nothofagus nervosa in scanning electron microscope. A and B groups of pollen grains, C and D polar view, equatorial view E, F colpo (c) and microspinules (e). 
Cuadro 3. Mediciones del polen de Nothofagus nervosa en vista polar $(\mu \mathrm{m})$. Se indica índice del área polar $(\mathrm{A} / \mathrm{L})$.

Measurements of Nothofagus nervosa pollen in polar view (microns). Index polar area (A/L) is indicated.

\begin{tabular}{|c|c|c|c|c|c|c|c|c|c|c|}
\hline \multirow{2}{*}{$\begin{array}{l}\text { Código } \\
\text { árboles }\end{array}$} & \multicolumn{5}{|c|}{ Ancho (A) } & \multicolumn{4}{|c|}{ Largo (L) } & \multirow{2}{*}{$\frac{\mathrm{A} / \mathrm{L}}{\bar{x}^{\mathrm{c}}}$} \\
\hline & $\mathrm{n}$ & $\bar{x}^{\mathrm{a}}$ & $\sigma$ & Mínimo & Máximo & $\bar{x}^{\mathrm{b}}$ & $\sigma$ & Mínimo & Máximo & \\
\hline 19 & 49 & $43,34 \mathrm{a}$ & 2,52 & 38,0 & 49,5 & $47,57 \mathrm{a}$ & 2,81 & 41,3 & 55,6 & $0,91 \mathrm{a}$ \\
\hline 11 & 52 & $44,20 \mathrm{a}$ & 2,50 & 39,3 & 53,1 & 46,98 a & 2,69 & 40,8 & 59,8 & $0,94 \mathrm{a}$ \\
\hline 21 & 48 & $45,60 \mathrm{~b}$ & 2,66 & 38,6 & 52,9 & $49,55 \mathrm{~b}$ & 2,61 & 44,2 & 56,0 & $0,92 \mathrm{a}$ \\
\hline 30 & 52 & $46,93 \mathrm{bc}$ & 2,26 & 44,1 & 51,8 & $49,50 \mathrm{ab}$ & 2,79 & 44,1 & 54,1 & $0,95 \mathrm{a}$ \\
\hline 26 & 43 & $49,34 \mathrm{c}$ & 1,26 & 47,8 & 51,1 & $53,23 \mathrm{~b}$ & 2,93 & 50,6 & 59,8 & $0,93 \mathrm{a}$ \\
\hline 38 & 72 & $49,79 \mathrm{c}$ & 3,48 & 40,8 & 60,7 & $53,31 \mathrm{~b}$ & 2,73 & 48,6 & 61,0 & $0,93 \mathrm{a}$ \\
\hline $\bar{x}$ & 52,7 & 46,53 & 2,45 & - & - & 50,02 & 2,76 & - & - & 0,93 \\
\hline
\end{tabular}

a. Letras diferentes indican medias diferentes, prueba de Kruskal Wallis $\mathrm{H}=119,35, P \leq 0,000$.

b. Letras diferentes indican medias diferentes, prueba de Kruskal Wallis $\mathrm{H}=66,99, P \leq 0,0000$.

c. Letras iguales indican medias iguales, prueba de Kruskal Wallis $\mathrm{H}=0,0000, P=1,000$.

Cuadro 4. Porcentaje de granos de polen por tipo de aperturas (\%) en Nothofagus nervosa.

Percentage of pollen grains per type of apertures (\%) in Nothofagus nervosa.

\begin{tabular}{|c|c|c|c|c|c|}
\hline \multirow{2}{*}{$\begin{array}{l}\text { Código } \\
\text { árboles }\end{array}$} & \multirow[b]{2}{*}{$\mathrm{n}$} & \multicolumn{4}{|c|}{ Número de aperturas } \\
\hline & & 6 & 7 & 8 & 9 \\
\hline 11 & 100 & 4,00 & 69,00 & 27,00 & 0,00 \\
\hline 19 & 64 & 20,65 & 60,94 & 15,87 & 3,13 \\
\hline 21 & 153 & 0,00 & 46,41 & 51,63 & 1,96 \\
\hline 26 & 71 & 7,04 & 56,34 & 33,80 & 2,82 \\
\hline 30 & 106 & 0,00 & 59,43 & 39,62 & 0,94 \\
\hline 38 & 90 & 2,22 & 55,56 & 42,22 & 0,00 \\
\hline $\bar{x}$ & - & 5,98 & 55,73 & 36,63 & 1,77 \\
\hline
\end{tabular}

dia para la especie de 65,08 $( \pm 8,8)$ (figura 3A). Los valores de germinación in vivo fueron altos en los individuos analizados, oscilando entre 77 y $98 \%$ (figura 3B). Los valores de viabilidad del polen, mediante el método de tinción fueron superiores a los de germinación in vitro, para todos los individuos analizados, estos oscilaron entre 70,5 y $99,3 \%( \pm 9,49-0,55)$, con una media de $89,5 \%( \pm 4,78)$ (figura $3 \mathrm{C}$ ). Se calculó una fuerte correlación entre la viabilidad calculada mediante germinación in vivo y germinación in vitro $(\mathrm{r}=0,91, P=0,004)$. En contraste, no hubo correlación entre los valores calculados mediante germinación in vivo y el método de tinción $(P=0,34)$ (figura $3 \mathrm{D})$.

La longevidad, en polen almacenado a temperatura ambiente, medida mediante germinación in vitro, fue has- ta de ocho días, aunque en bajos porcentajes. La mayor pérdida de viabilidad ocurrió al cuarto día de evaluación, puesto que la germinación del polen disminuyó a menos del $20 \%$ en la mayoría de los árboles analizados. La pérdida de viabilidad fue directamente proporcional a los días de almacenaje ( $\mathrm{r}=-0,92 ; P<0,00001)$ (figura 4$)$. El polen de los diferentes individuos analizados perdió la viabilidad rápidamente sin considerar su germinabilidad inicial; no obstante hubo individuos en los que la viabilidad del polen no disminuyó significativamente al segundo día de evaluación, aunque incluso para estos la viabilidad del polen cayó a menos del $20 \%$ al cuarto día.

\section{DISCUSIÓN}

Según las observaciones y mediciones presentadas en este documento, el polen de $N$. nervosa corresponde al tipo menziessii, apoyando la clasificación anteriormente establecida por varios investigadores (Cranwell 1939, Auer et al. 1955, Veblen et al. 1996). Igualmente, Heusser (1971) lo describió dentro del tipo polínico obliqua, que, como él menciona, corresponde al tipo menziessii que en un inicio estableció Cranwell (1939). Sin embargo, Wang (2000) lo clasificó dentro del tipo polínico fusca, con tamaños más pequeños que los reportados hasta ese momento. Este autor coloca $N$. nervosa en este grupo, aunque también coloca Nothofagus procera (Poepp. et Endl.) Oerst en el tipo polínico menziesii, siendo conocido que estos dos nombres son sinonimias.

El tamaño de los granos de polen, varía entre los individuos de $N$. nervosa evaluados, presentan diferencias significativas tanto en el eje polar medido en vista ecuatorial como en ancho medido en vista polar. De acuerdo a la escala propuesta por Erdtman (1952), los granos de 
polen son de tamaño mediano a grande. Según la relación: eje polar/eje ecuatorial, es de forma sub oblada a oblada esferoidal, lo que concuerda con descripciones realizadas por investigadores como Heusser (1971) y Dettman et al. (1990), quienes documentaron que estos granos de polen son de forma oblada a suboblada. En cuanto al tamaño, en vista ecuatorial, los valores obtenidos aquí (35-54 x 40-60 $\mu \mathrm{m})$ son similares a los reportados por Heusser (41-46 x 48-67 $\mu \mathrm{m}$ ), pero significativamente más altos a los repor- tados por Wang (2000). Es probable que la variabilidad intraespecífica en la característica tamaño del polen, pueda utilizarse para apoyar la identificación molecular de los clones del huerto Huillilemu.

En términos generales, se observan entre seis y nueve aperturas tipo colpos, concordando con la descripción realizada por Heusser (1971), quien documentó que estos granos de polen pueden tener de seis a ocho colpos, mientras Wang (2000) describe este tipo de polen con seis a nueve
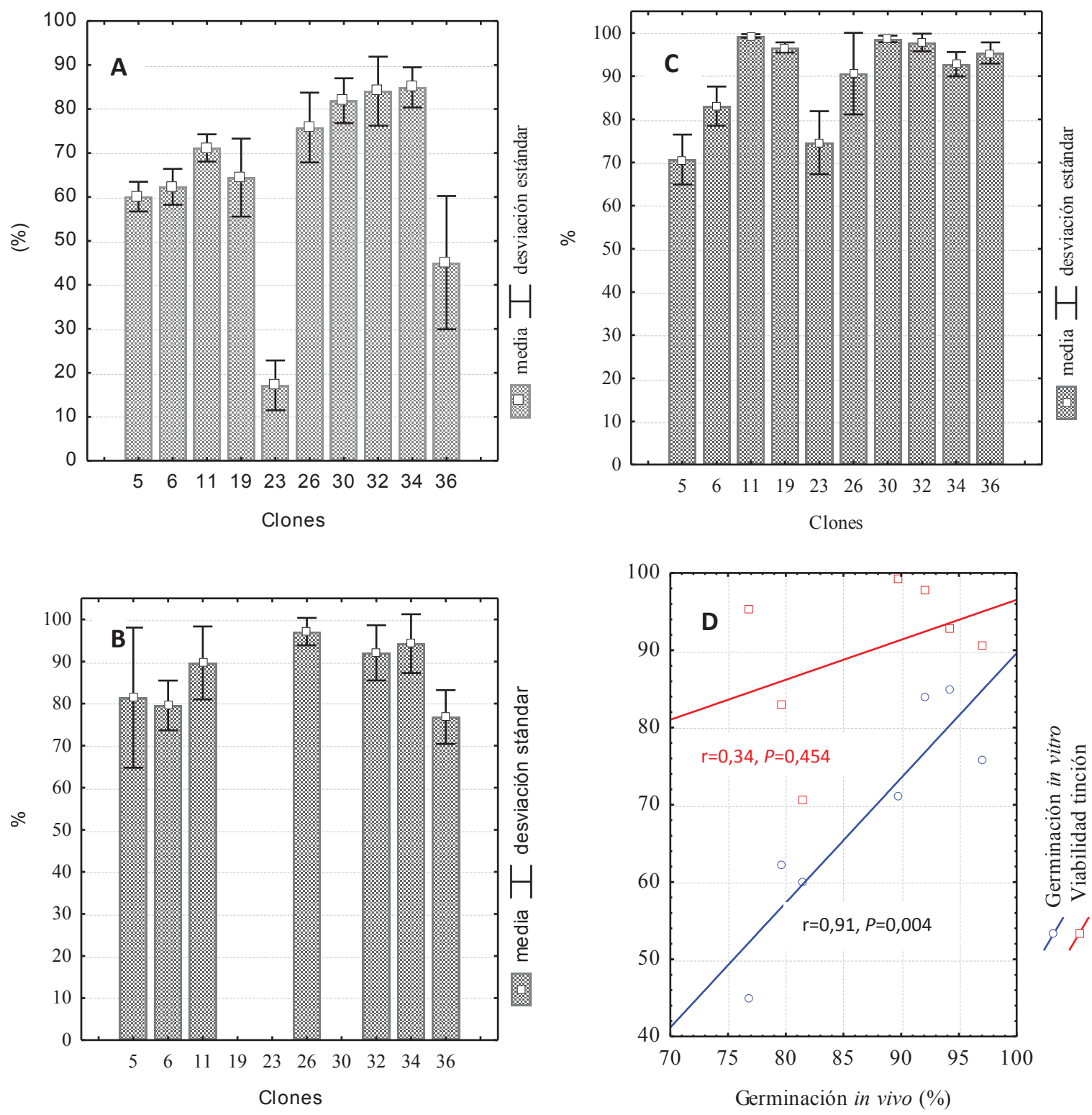

Figura 3. Viabilidad del polen de diez clones de Nothofagus nervosa mediante germinación in vitro (A) KW-H=66,83, germinación in vivo (B) $\mathrm{KW}-\mathrm{H}=0$ y tinción con p-fenilendiamina (C) $\mathrm{KW}-\mathrm{H}=74,69$, en el huerto semillero clonal "Huillilemu", 2011. Se encontraron diferencias significativas entre clones según la prueba de Kruskal Wallis $(P \leq 0,0001)$. Ecuación de regresión Germinación in vivoGerminación in vitro: $\mathrm{y}=-542,341+5,884 * \mathrm{x}$.

Pollen viability of ten clones of Nothofagus nervosa using in vitro germination (A) $\mathrm{KW}-\mathrm{H}=66.83$, germination in vivo (B) $\mathrm{KW}-\mathrm{H}=0$ and stained with p-phenylenediamine (C) KW-H $=74.69$ in clonal seed orchard "Huillilemu", 2011. Significant differences among clones were found according to the Kruskal Wallis test $(P<0.000)$. Regression equation germination in vivo-in vitro: $\mathrm{y}=-542.341+5.884 \mathrm{x}$. 


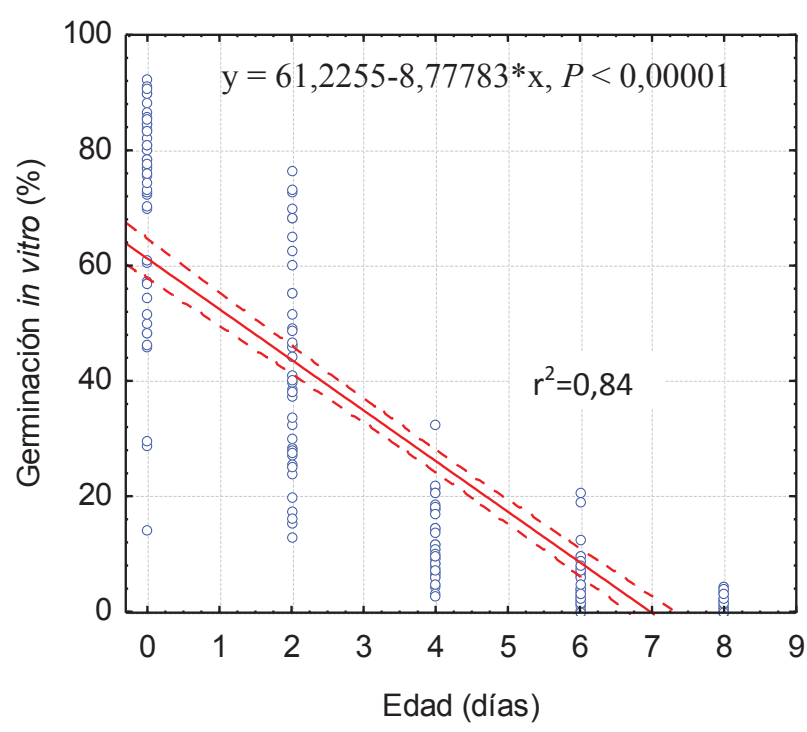

Figura 4. Longevidad del polen evaluado mediante germinación in vitro a temperatura ambiente (A), huerto semillero Huillilemu, 2012. Las líneas punteadas indican el intervalo de confianza $(P=0,95)$.

Longevity of pollen evaluated by in vitro germination at room temperature (A), seed orchard "Huillilemu" 2012. Dotted lines indicate the confidence interval ( 0.95$)$.

colpos. Existe diferencia entre clones en cuanto al número de aperturas presentes en los granos de polen, por ejemplo, para el clon 19 se calculó un $20 \%$ de granos de polen con seis aperturas, mientras en los clones 21 y 30 no se encontró granos de polen con este número de aperturas. Los granos de polen con nueve aperturas son los menos frecuentes e incluso no se presentan en los clones 11 y 38 . En cambio, hay mayor porcentaje de granos de polen con siete y nueve aperturas. En cinco de los seis clones evaluados, son más frecuentes los granos de polen con siete aperturas, que se encontraron en un $55,73 \%$, mientras que un $36,63 \%$ de los granos revisados tienen ocho aperturas. Al parecer el número de aperturas en los granos de polen no tiene relación con su origen altitudinal, pues independientemente de este, todos los clones evaluados presentan la mayor parte de sus granos de polen con siete aperturas a excepción del clon 21 que tiene aproximadamente el mismo porcentaje de granos de polen con siete y ocho aperturas $(46,41$ y $51,63 \%$, respectivamente).

Los granos de polen de esta especie, son semejantes a los de otras especies reunidas dentro de este tipo polínico, como Nothofagus obliqua (Mirb.) Oerst. y Nothofagus glauca (Phil.) Krasser, según la descripción realizada por Heusser (1971); quien además señala que el tamaño y número de aperturas en los granos de polen, varía considerablemente en cada población de las especies de la familia Nothofagaceae. Esto hace suponer que el diferente origen altitudinal de los individuos evaluados, podría ser la causa de variación en el tamaño y forma en los granos de polen, aunque también se debe tomar en cuenta la historia de vida de cada individuo, en vista de las posibilidades de hibridación entre las especies del género Nothofagus (Donoso y Atienza 1984, Donoso et al. 1990, Gallo et al. 2000. Stecconi et al. 2004).

El polen de $N$. nervosa presenta valores altos de viabilidad mediante los tres métodos evaluados, por lo que la viabilidad del polen no se debería considerar como un factor limitante en la producción de semillas en el huerto Huillilemu. No existen otros trabajos relacionados a la viabilidad del polen de esta especie, pero sí para otras especies de la misma familia (Nothofagus antarctica G. Forst, Nothofagus obliqua, Nothofagus dombeyi (Mirb.) Blume y Nothofagus betuloides (Mirb.) Blume), en los que se encontró una germinación promedio, para el polen de árboles adultos, entre 68 y $84 \%$ (Báez et al. 2002), similar a lo reportado aquí para $N$. nervosa $(65,08 \pm 8,8)$.

En el marco de programas de mejoramiento genético es importante conocer la viabilidad inicial del polen y la forma idónea de evaluarla, para planificar eficientemente los planes de cruzamiento controlado y suplementación polínica. No sería recomendable utilizar polen de individuos que presenten baja viabilidad polínica. Este estudio no da indicios de que la viabilidad polínica tenga control genético, pues la investigación es una aproximación biológica y no genética a las diferencias del polen. De acuerdo a observaciones realizadas en el desarrollo de esta investigación, se recomienda que el polen se evalúe en los diferentes individuos, cada vez que se vaya a utilizar, pues puede variar en diferentes temporadas en un mismo individuo, de acuerdo a las condiciones climáticas, e incluso dentro de la misma temporada si la muestra se toma en diferentes estados de maduración floral masculina o a diferentes horas del día. Por lo mismo, las muestras de polen utilizadas fueron extraídas de flores colectadas en un mismo estado fenológico y bajo las mismas condiciones climáticas.

A partir de los resultados, se aprecia una fuerte correlación entre la viabilidad calculada mediante germinación in vivo y germinación in vitro, y débil entre la viabilidad obtenida mediante germinación in vivo y el método indirecto de tinción. Estos resultados sirven de antecedentes para recomendar la utilización del método germinación in vitro para estudios de viabilidad inicial del polen y para realizar monitoreo del polen durante el almacenamiento.

A raíz de este trabajo, se observa que el polen recién liberado se tiñe de negro rápidamente, utilizando el reactivo de p-fenilendiamina, en cambio el polen almacenado por varios días o semanas se tiñe de un color cada vez más pálido, hasta el momento que prácticamente no se tiñe. Con esto se deduce que el polen estando vivo, se tiñe de un color pardo claro, resultado que no se relaciona con su capacidad o vigor para germinar. Aunque, dado el uso práctico que tiene el método de tinción (reactivo p-fenilendiamina), sería recomendable para futuros trabajos, realizar una cartilla donde se relacione el color de los granos de polen con 
el porcentaje de germinación obtenido en una muestra determinada.

Por otro lado, la longevidad del polen de $N$. nervosa, a temperatura ambiente $\left( \pm 20{ }^{\circ} \mathrm{C} \mathrm{y} \pm 40 \% \mathrm{HR}\right)$ disminuye tan rápidamente, que a los cuatro días había menos del 20 $\%$ de polen viable para los diferentes individuos evaluados; lo cual, según Heslop-Harrison y Heslop-Harrison (1985) y Lisci et al. (1994), estaría relacionado con la pérdida de agua y un prolongado estado de deshidratación. La corta longevidad del polen de $N$. nervosa podría ser una característica propia de la especie, o tal vez la diferencia notoria con respecto a los Nothofagus spp. evaluados por Báez et al. (2002) (según quienes el polen se mantuvo vivo por tres meses) podría deberse a que estos autores realizaron el almacenamiento del polen a $4{ }^{\circ} \mathrm{C}$ y para evaluar la longevidad utilizaron la tinción de Alexander. Pues, de acuerdo a Rodríguez-Riano y Dafni (2000) varios reactivos, como la tinción de Alexander, tiñen polen incluso estando muerto, dando lugar a falsos positivos.

El polen de $N$. nervosa es liberado al aire durante cuatro a ocho días después de la dehiscencia de las anteras, según las condiciones climáticas predominantes (García et al. 2013). Esta rápida liberación, la corta longevidad del polen y el desfase en la fenología de los árboles, registrado por García et al. (2013), limitan el éxito de los planes de cruzamiento cuando no se ha realizado un proceso de almacenamiento ad hoc. La determinación de los requisitos fisiológicos para un adecuado manejo del polen, será valiosa en los programas de mejoramiento genético con el objetivo de mejorar la producción de semillas de cruzamientos controlados.

\section{CONCLUSIONES}

Los resultados obtenidos son consistentes con las caracterizaciones morfológicas realizadas para las especies del tipo polínico menziessi del subgénero Lophozonia. Los individuos analizados presentan uniformidad en los caracteres morfológicos de forma, simetría, tipo de aperturas, pero hay diferencias en el tamaño que podrían atribuirse a las posibles diferencias en la historia de vida de los genotipos evaluados.

La viabilidad inicial del polen, varía en los diferentes individuos analizados; mediante germinación in vitro fue relativamente alta, a excepción de los árboles 23 y 36 que presentan una menor germinación. Se observa fuerte correlación entre la germinación in vitro y la germinación in vivo, por lo que se recomienda este método para evaluar la viabilidad inicial del polen y realizar monitoreo durante el almacenamiento.

La viabilidad del polen decrece rápidamente con el tiempo, bajo las condiciones de almacenamiento evaluadas, por lo que es necesario realizar ensayos que involucren diferentes condiciones de secado, humedad relativa y temperaturas, para el almacenamiento de polen de esta especie a mediano o largo plazo.

\section{AGRADECIMIENTOS}

Los autores agradecen al proyecto FONDEF D10i1149 por el financiamiento para realizar esta investigación, al Sr. Cristian Montalva Retamal por su ayuda en el manejo del programa Motic Images Plus 2.0 utilizado para realizar las mediciones de los granos de polen, al Sr. Eladio Rojas Pérez por permitir el uso de equipo y reactivos para las capturas fotográficas en el Laboratorio Regional SAG Osorno, al Sr. Ricardo Silva por las imágenes en microscopio electrónico de barrido, a la Srta. Érica Pérez por su colaboración en el laboratorio. Luz García también agradece al proyecto MECESUP UCO0702 y a la Secretaría de Ciencia y Tecnología del Ecuador por becas de estudios doctorales durante la realización de este estudio.

\section{REFERENCIAS}

Auer V, M Salmi, K Salminen. 1955. Pollen and spore types of Fuego-Patagonia. Annales Academiae Scientiarum Fennicae III. Geologica-Geographica 43: 1-10.

Báez P, M Riveros, C Lehnebach. 2002. Viability and longevity of pollen of Nothofagus species in South Chile. New Zealand Journal of Botany 40(4): 671- 678.

Bellani L, E Paoletti. 1992. New type of damage to Quercus ilex: pollen germination and hydration ability. European Journal of Forest Pathology 22: 284-290.

Cecich R. 1997. Pollen tube growth in Quercus. Forest Science 43: 140 - 146.

Cranwell L. 1939. Southern beech pollens. Records of the Auckland Institute Museum 2(4): 175-96.

Dafni A, D Firmage. 2000. Pollen viability and longevity: practical, ecological and evolutionary implications. Plant Systematics and Evolution 222: 113-132.

Dettmann M, D Pocknall, E Romero, M Zamaloa. 1990. Nothofagidites Erdman ex Potonie 1960: a catalogue of species with notes on the paleogeographic distribution of Nothofagus BL (Southern Beech). New Zealand Geological Survey Palaeontological Bulletin 60: 1-79.

Donoso C, J Atienza. 1984. Hibridación natural entre Nothofagus betuloides (Mirb.) Oerst. y Nothofagus nitida (Phil.) Krasser. Medio Ambiente 7: 9-16.

Donoso P, C Donoso, M Azpiculeta. 2006. Nothofagus nervosa (Phil.) Dim. et Mil. In Donoso C ed. Las especies arbóreas de los bosques templados de Chile y Argentina. Autoecología. Valdivia, Chile. Ediciones Marisa Cuneo. p. 448-461.

Donoso C, J Morales, M Romero. 1990. Hibridación natural entre roble (Nothofagus obliqua (Mirb.) Oerst.) y raulí (Nothofagus alpina (Poepp. et Endl.) Oerst.) en bosques del sur de Chile. Revista Chilena de Historia Natural 63: 49-60.

Erdtman G. 1952. Pollen Morphology and Plant Taxonomy: Angiosperms. Stockholm, Sweden. Almqvist and Wiksell. 553 p.

Gallo L, P Marchelli, M Azpilicueta, P Crego. 2006. El uso de marcadores genéticos en el género Nothofagus con especial referencia en raulí y roble. Bosque 27(1): 3-15.

Gallo L, P Maxhelli y P Crego. 2000. Variación genética en poblaciones y progenies de Raulí en Argentina. III. Dinámica de hibridación interespecífica entre raulí y roble pellín. In 
Ipinza R, B Gutiérrez, V Emhart eds. Domesticación y mejora genética de raulí y roble. Valdivia, Chile. p. 403-418.

García L, F Droppelmann, M Rivero. 2013. Morfología y fenología floral de Nothofagus alpina (Nothofagaceae) en un huerto semillero clonal en la región de Los Ríos, Chile. Bosque 34(2): 221-231.

Heslop-Harrison J, Y Heslop-Harrison. 1985. Germination of stress tolerant Eucalyptus pollen. Journal of Cell Science 73: 135-157.

Heusser C. 1971. Pollen and spores of Chile. Modern types of the Pteridophyta, Gymnospermae and Angiospermae. Tucson, Arizona. University of Arizona Press. 167 p.

Hill RS, GJ Jordan. 1993. The evolutionary history of Nothofagus (Nothofagaceae). Australian Systematic Botany 6: 111-126.

Lisci M, C Tanda, E Pacini. 1994. Pollination ecophysiology of Mercurialis annua L. (Euphorbiaceae) an anemophilous species flowering all year round. Annals of Botany 74: 125-135.

Manos P. 1997. Systematics of Nothofagus (Nothofagaceae) based on rDNA spacer sequences (ITS): taxonomic congruence with morphology and plastid sequences. American Journal of Botany 84(9): 1137-1155.

Pérez C, R Ponce (eds). 2012. Guía para el uso de la información agroclimática en el manejo de cultivos y frutales. Santiago, Chile. Instituto de Investigaciones agropecuarias INIA, Fundación de Desarrollo Frutícola FDF y Unidad Nacional de Emergencias Agrícolas y Gestión del Riesgo Agroclimático UNEA. 104 p.
Riveros M, M Paredes, M Rosas, E Cárdenas, J Armesto, M Arroyo. 1995. Reproductive biology in species of the genus Nothofagus. Environmental and Experimental Botany 35: 519-524.

Rodríguez-Riano T, A Dafni. 2000. A new procedure to asses pollen viability. Sexual Plant Reproduction 12: 241-244.

Sabatier Y, M Azpilicueta, P Marchelli, M González-Peñalba, L Lozano, L García. 2011. Distribución natural de Nothofagus alpina y Nothofagus obliqua (Nothofagaceae) en Argentina, dos especies de primera importancia forestal de los bosques templados norpatagónicos. Boletín de la Sociedad Argentina de Botánica 46 (1-2): 131-138.

Shivanna K, N Rangaswamy. 1992. Pollen biology: a laboratory manual. New York, USA. Springer. 118 p.

Stecconi M, P Marchelli, J Puntieri, P Picca, L Gallo. 2004. Natural hybridation between a deciduous (Nothofagus antarctica, Nothofagaceae) and an evergreen ( $N$. dombeyi) forest tree species: evidence from morphological and isoenzymatic traits. Annals of Botany 94 (6): 775-786.

Torres C, J Puntieri. 2013. Pollination and self-interference in Nothofagus. Flora - Morphology, Distribution, Functional Ecology of Plants 208 (7): 412-419.

Veblen T, R Hill and J Read (eds.). 1996. The Ecology and Biogeography of Nothofagus Forests. New Haven, USA. Yale University Press. 403 p.

Wang P, F Pu, Z Zhen. 2000. Pollen morphology of the genus Nothofagus and its taxonomic significance. Acta Phytotaxonomica Sinica 38 (5): 352-461. 